Provided for non-commercial research and education use. Not for reproduction, distribution or commercial use.

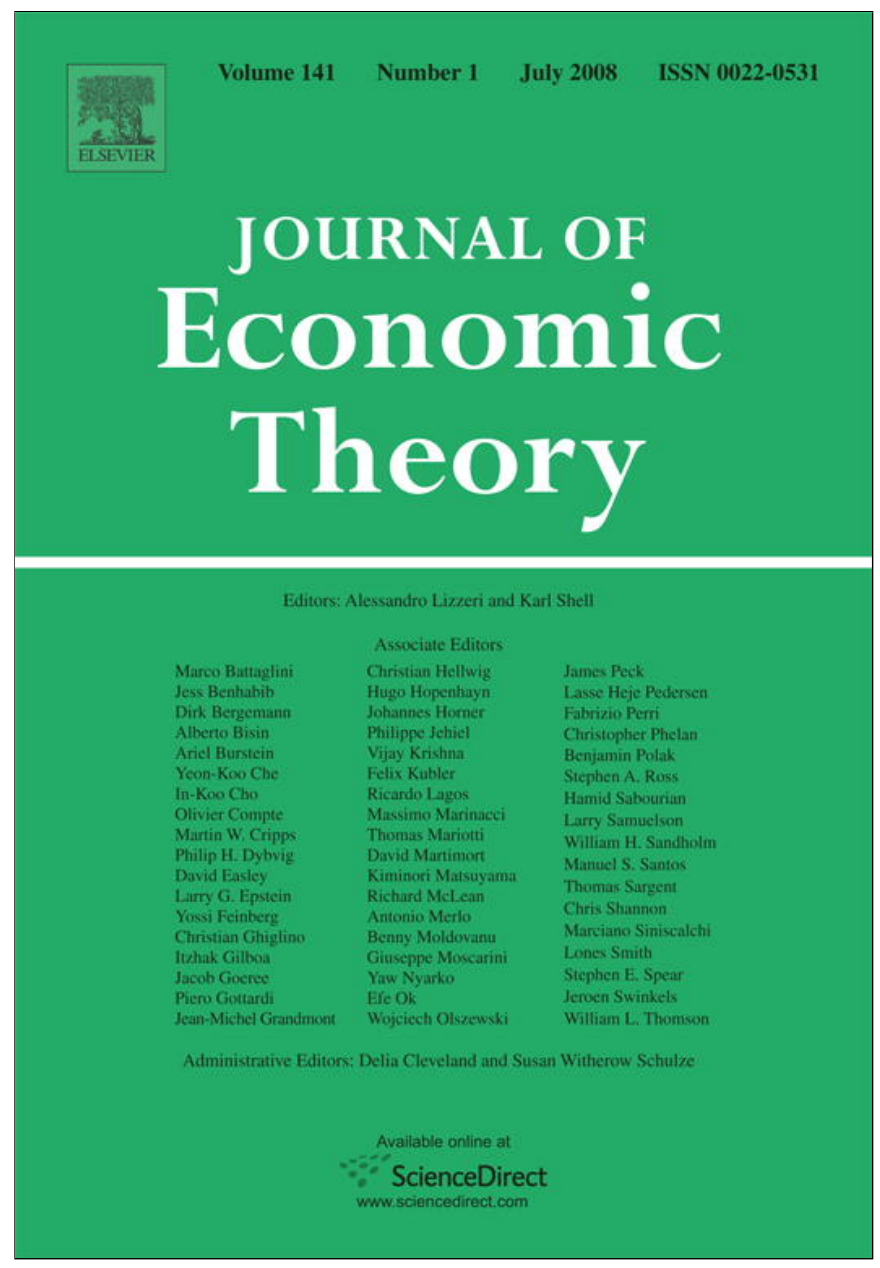

This article appeared in a journal published by Elsevier. The attached copy is furnished to the author for internal non-commercial research and education use, including for instruction at the authors institution and sharing with colleagues.

Other uses, including reproduction and distribution, or selling or licensing copies, or posting to personal, institutional or third party websites are prohibited.

In most cases authors are permitted to post their version of the article (e.g. in Word or Tex form) to their personal website or institutional repository. Authors requiring further information regarding Elsevier's archiving and manuscript policies are encouraged to visit:

http://www.elsevier.com/copyright 


\section{JOURNAL OF}

Economic Theory

www.elsevier.com/locate/jet

\title{
Competing auctions with endogenous quantities
}

\author{
Benny Moldovanu ${ }^{\mathrm{a}, *}$, Aner Sela $^{\mathrm{b}}$, Xianwen Shi ${ }^{\mathrm{c}}$ \\ ${ }^{a}$ Department of Economics, University of Bonn, Lennestr. 37, 53113 Bonn, Germany \\ ${ }^{\mathrm{b}}$ Department of Economics, Ben Gurion University, Beer Sheva 84105, Israel \\ ${ }^{\mathrm{c}}$ Department of Economics, University of Toronto, 100 St. George Street, Toronto, Ont., Canada M5S 3G3
}

Received 7 February 2007; received in revised form 11 July 2007; final version received 11 September 2007

Available online 5 October 2007

\begin{abstract}
Two sellers decide on their discrete supply of a homogenous good. There is a finite number of buyers with unit demand and privately known valuations. In the first model, there is a centralized market place where a uniform auction takes place. In the second, there are two distinct auction sites, each with one seller, and buyers decide where to bid. Using the theory of potential games, we show that in the one-site auction model there is always an equilibrium in pure-strategies. In contrast, if the distribution of buyers values has an increasing failure rate, and if the marginal cost of production is relatively low, there is no pure-strategy equilibrium where both sellers make positive profits in the competing sites model. We also identify conditions under which an equilibrium with a unique active site exists. We deal with the finite and discrete models by using several results about order statistics developed by Richard Barlow and Frank Proschan [R. Barlow, F. Proschan, Mathematical Theory of Reliability, Wiley, New York, 1965; R. Barlow, F. Proschan, Inequalities for linear combinations of order statistics from restricted families, Ann. Math. Statist. 37 (1966) 1593-1601; R. Barlow, F. Proschan, Statistical Theory of Reliability and Life Testing, McArdle Press, Silver Spring, 1975].
\end{abstract}

(C) 2007 Elsevier Inc. All rights reserved.

JEL classification: D43; D44; D82; L13

Keywords: Competing auctions; Order statistics

\section{Introduction}

We study models where two sellers simultaneously decide on their (discrete) supply of a homogenous good, and where there is a finite, not necessarily large, number of buyers who have

\footnotetext{
* Corresponding author. Fax: +49228737940.

E-mail addresses: mold@uni-bonn.de (B. Moldovanu), anersela@bgu.ac.il (A. Sela), xianwen.shi@utoronto.ca (X. Shi).
} 
unit demand and privately known valuations. In the first model, the sellers bring their supply to a centralized market place where a uniform-price auction takes place (the price equals the highest losing bid). Thus, by the intrinsic rules of the mechanism, all sold units command the same price. In the second model, there are two distinct auction sites, each with one seller. Before observing their value, but after observing the respective supplied quantities at each site, buyers decide which auction to attend (again, each auction is a uniform-price one). In principle, each of the two auctions may have its own equilibrium price.

The present paper has several purposes:

1. Our main results (that easily generalize to any number of sellers/auction sites) shed some light on the conditions leading to either the emergence of one dominant marketplace, or to the coexistence of several competing sites. In the one auction-site model there is always an (almost symmetric) equilibrium in pure strategies. This equilibrium approximates the Cournot outcome as the number of buyers becomes large. In contrast, if the distribution of buyers values has an increasing failure rate (IFR) (a condition assumed in most of the literature, which implies that marginal revenue is decreasing), and if the marginal cost of production is relatively low, there is no pure-strategy equilibrium where both sellers make positive profits in the competing sites model. In other words, two distinct auction sites cannot coexist in equilibrium under these conditions. Coexistence becomes possible only if the marginal cost of production is sufficiently high. We also identify conditions under which an equilibrium with a unique active site exists.

2. We revisit several classical scenarios in monopoly and oligopoly theory. These theories discard the standard assumption of competitive analysis concerning the large number of producing firms (sellers), but they keep the assumption that the number of consumers (buyers) is large. Our model has both a small number of sellers with endogenous supply, and a small number of buyers. ${ }^{1}$ This framework is appealing in many situations, in particular in markets for inputs where both upstream and downstream markets are relatively concentrated oligopolies.

3. Finally, we introduce to the analysis of competing auctions several very useful tools from the literature on order statistics [5-7]. These tools allow us to consistently deal with finite and discrete models in which each agent's decision may have a significant impact on the outcome. Admittedly, our economic models are rather simplistic, and tailored to facilitate the use of these statistical tools. Nevertheless, we believe that many more applications are possible. ${ }^{2}$

One way to unify the two main models of the paper is in terms of buyers' switching cost. If the switching cost is high, that is, once buyers decide to place a bid in one auction they cannot switch to the other, we obtain the model with two competing auction sites. If the switching cost is low, then an ascending clock auction a la Demange-Gale-Sotomayor (this is a variant with money on Gale-Shapley's deferred acceptance algorithm) where buyers shift demand to the cheaper unit, leads to an efficient outcome (conditional on supplied quantities) [13]. This outcome is equivalent to the one of the uniform-price auction in the single site model-in particular, all units are sold at a single market price.

Viewed in this perspective, the competing auctions model is one where sellers compete for market share in the quantity-setting stage, i.e., they compete for the buyers that will become locked-in at the second stage (see Klemperer, and Klemperer and Meyer, for an early analysis of

\footnotetext{
${ }^{1}$ The parallels between a single-good auction theory and monopoly are well-known [9,10]. Hansen studies a model where sellers compete for the right to become the sole supplier to a unique buyer whose purchase depends on the winning bid [18].

${ }^{2}$ For applications in other contexts, see [19,29].
} 
the forces at play in such models [20,21]). "Lock-ins" appear in many settings and have a variety of reasons such as transaction costs associated with operating via several technologically different platforms/clearing houses, uncertainty about the quality/quantity offered at other marketplaces, "loyalty" contracts with operators, ex ante market specific investments, and various forms of network externalities.

On the other hand, a model with a centralized auction site and with endogenous supply is better suited for the study of some modern markets where all transactions are executed at market price, i.e., order driven periodic auctions in financial markets, or markets for inputs such as electricity or gas. ${ }^{3}$ At online auction sites such as eBay there are often several simultaneous auctions for identical commodities (e.g., CPUs). Anwar et al. empirically study such parallel auctions and find that a significant proportion of bidders are indeed active across the competing auctions: they repeatedly place bids in the auctions with the lowest standing bid, and prices tended to be uniform across auctions [3].

In contrast to our paper, most of the papers in the relatively thin literature on competing auctions consider models with several sellers endowed with a single unit of a homogenous good, and with several buyers with unit demand who decide which auction to attend. Thus, total demand and supply are fixed exogenously, whereas total supply is endogenous in our models. Most of these papers use some kind of "large market assumption" for their main results (i.e., individual agents ignore the effect of their own actions on prices) while such an assumption is not needed here. It is not always clear whether such assumptions are consistent with a limit of equilibria arising in small markets of increased size.

Peters and Severinov consider several sellers who set reserve prices at their own auction sites and then conduct second price auctions. A large number of buyers with unit demand can move among auction sites [35]. The main consequence of this freedom (i.e., absence of switching costs) is that there exists an efficient equilibrium (conditional on reserve prices). This is consistent with the insight obtained from the models that mimic features of the Gale-Shapley deferred acceptance algorithm (such as our model of competing sellers at a unique auction site) and should be contrasted with earlier models such as McAfee [28] ${ }^{4}$ or Peters and Severinov where buyers can place only one bid, thus leading to an inefficiency stemming from the coordination problem [34].

The role of a unique marketplace is also the theme of Moreno and Ubeda who introduce an explicit element of price formation in the traditional Cournot oligopoly story (while keeping the assumption that there is a large number of buyers) [32]. Their starting point is the classical paper by Kreps and Scheinkman who study a two stage game, with capacity choice in the first stage, and Bertrand competition in the second stage [23]. Kreps and Scheinkman show that the unique equilibrium coincides with the Cournot outcome. Moreno and Ubeda look at the two stage model where firms first choose capacity and then set reservation prices at which they are willing to sell their entire capacity. The ensuing supply function is used to clear the market [32]. The main difference to the Kreps and Scheinkman analysis is that all supply is sold at market price whereas,

\footnotetext{
${ }^{3}$ Lengwiler, Back and Zender and McAdams analyze endogenous supply in monopolistic models where the seller adjusts supply after seeing the realization of demand, and where bidders demand several units [24,4,27]. LiCalzi and Pavan allow the monopolist to ex ante commit to a supply function [25]. This is in the spirit of Klemperer and Meyer who study supply-function equilibria in oligopoly with demand uncertainty [21].

${ }^{4} \mathrm{He}$ considers several sellers offering auctions at fixed sites (one seller for each site). His analysis assumes that the economy is large, and therefore it ignores the fact that a change in one seller's mechanism affects the distribution of buyers at his own and at other sites.
} 
in Kreps and Scheinkman's model each firm charges its own price. This allows them to avoid several difficulties appearing in the Kreps-Scheinkman model. ${ }^{5}$

If we introduce reserve prices in our model of competing sellers at one auction site, and if we let the number of buyers go to infinity (which is the standard assumption in the literature) it can be shown that, if quantity is optimally adjusted to the number of buyers, the marginal gain from a reserve price goes to zero. ${ }^{6}$ The limit outcome is then the classical Cournot one-corresponding to the main result of Moreno and Ubeda [32].

An interesting strand that allows for both a small number of buyers and a small number of sellers is the literature on the so-called strategic market games, following the pioneering work by Shapley and Shubik [36]. In these models both buyers and sellers bid quantities, and transactions are cleared at a price equal to the ratio of demand to supply (thus, commodities are allocated in proportion to bids). Almost the entire literature assumes that the number of trading sites (or "posts") is the same as the number of traded commodities. In other words, there is a unique trading site for each traded commodity where all demand and supply of that commodity are cleared. A notable exception is Koutsougeras who allows for two separate trading sites for the same good. In his model traders can send quantity bids to either one, or to both sites. He shows via an example that the "Law of One Price" need not hold in equilibrium. Different prices open up arbitrage opportunities for agents who can shift their demand/supply (which seems inconsistent with the equilibrium idea), but these opportunities disappear when individual agents (who are relatively speaking, "large") try to take advantage of them [22]. Such phenomena, which also appear in our model, nicely illustrate the caution that needs to be employed when dealing with models with a small number of traders. In particular, market structure may matter a lot: in our model, even mere equilibrium existence is affected by it.

Our model of competing auction sites with two possibly distinct prices is related to that of Ellison, Fudenberg and Mobius [16]. These authors study a situation where both sellers and buyers (each with unit demand/supply) simultaneously choose one of two auction sites-this is an application of a more general framework developed by Ellison and Fudenberg [15]. Thus, both buyers and sellers are locked-in at their respective sites, and both total supply and demand in the market are fixed. Prices are determined by uniform-price auctions at each site (and thus by the ratio of buyers to sellers at each location) [15]. ${ }^{7}$ Their elegant analysis ignores the integer constraints, and some results hold only for markets with large numbers of traders. ${ }^{8}$ Ellison et al. focus on the conditions leading to the existence of equilibria where two auction sites of unequal size can coexist. ${ }^{9}$ Their solved examples involve the uniform and exponential distributions of values-thus both display an IFR. These coexistence results should be contrasted to ours under this assumption.

\footnotetext{
5 The K-S result critically depends on a particular rationing rule used when capacity is binding. Davidson and Deneckere show that the Cournot outcome cannot be the equilibrium of the two stage game for any other rationing rule. Moreover, for a wide range of capacity choices, the equilibrium at the price competition stage is in mixed strategies, leading to ex post regret [12].

6 This result is based on an analogous observation for a monopolistic firm that optimally adjusts quantity in response to a varying number of buyers. Since an increase (decrease) in quantity causes a decrease (increase) in price, a binding reserve price becomes superfluous as an instrument to control price. This should be contrasted to what happens if competing sellers have only one unit to sell [11].

${ }^{7}$ We could have also presented our model by assuming that: (1) every unit is owned by a different seller; (2) the operator of each auction site restricts entry of sellers in order to maximize total profit at the site.

${ }^{8}$ Anderson et al. retake this analysis while incorporating the integer constraints since ignoring them may lead to inconsistent results [2].

${ }^{9}$ Behringer introduces platform charges to this model and studies the structure of the ensuing equilibria [8].
} 
A main difference between the EFM model and ours is in timing: all their agents move simultaneously, whereas here buyers decide which auction to attend only after seeing the supplied quantities. We offer below an example that keeps the endogeneity in quantities but adopts the EFM assumption of simultaneous moves of both buyers and sellers. The example exhibits a multitude of (possibly asymmetric) equilibria. In our model, a seller can "poach" one bidder away from the other auction site by supplying one more unit. Under a general condition on the distribution of values (IFR), poaching increases profits. If all agents move simultaneously, poaching only increases supply and lowers profits.

Similar to our model, Monderer and Tenenholz [31] analyze competition among sellers over a set of buyers who decide which auction to attend. Each seller has one unit of the good in their model, and chooses a $k$-price auction where $k \in\{1,2, \ldots, K\}$. Using payoff-equivalence results, Monderer and Tenenholz characterize buyer-symmetric equilibria and determine which type of auction will be used as a function of the buyers' risk attitudes.

In a different vein, Neeman and Vulkan study the coexistence of two forms of trade: direct bilateral negotiations and centralized markets. ${ }^{10}$ In their model, buyers with a high willingness to pay and sellers with low costs prefer to trade through a centralized market. This leads to an unraveling of direct negotiations, so that ultimately, all traders opt for trading through the centralized market [33]. Their analysis uses a reduced form formulation and depends on the assumption that there are a large number of traders.

The present paper is organized as follows. In Section 2 we describe the basic model ingredients and introduce useful definitions and notation. In Section 3 we study the case of one auction site. In Section 3.1 we start with a monopolistic seller using a uniform-price auction. We focus on the optimal supplied quantity, and we show that it increases in the convexity of the distribution function of buyers' valuations. This allows us to derive optimal quantity estimates for large, nonparametric families of distributions (i.e., with monotone hazard rates, concave, convex). In Section 3.2 we remain in the single auction-site model, but add competition among sellers: each of two ex ante symmetric sellers provides the auctioneer with several units of a homogenous good, and the auctioneer sells the total quantity via a uniform-price auction. With a large number of buyers, this is equivalent to classical Cournot competition. Using the potential game approach [30] we prove the existence of pure-strategy Nash equilibria for any constant marginal cost. Moreover, we show that an "almost" symmetric equilibrium, where the supplied quantities differ by at most one unit, always exists. Finally, we use the above described estimates in the monopoly case to give estimates for the total quantity under competition.

In Section 4 we turn to the model with two distinct auction sites, each with one seller: First, the two sellers simultaneously choose the number of units supplied in their respective auctions. Before they learn their valuations, but after they observe the sellers' decisions, buyers choose which auction to attend. In the last stage, each buyer learns his valuation and submits a bid in his selected auction. In Section 4.1 we study the possibility of coexistence of two auction sites. A main result is that if the marginal production cost is low enough, and if the distribution of buyers' values has an increasing hazard rate then there is no pure-strategy equilibrium in which both sellers are active and make positive profits. The reason is that each seller continually increases supply in order to snatch buyers from the other site. A coexistence equilibrium is possible only if the marginal cost of production is high enough. Then, the unilateral incentives to increase supply are capped by the production cost. In Section 4.2 we study equilibria where only one auction site is active (i.e., the other seller prefers to stay out). Such an equilibrium is possible if the optimal

${ }^{10}$ Genesove [17] offers an insightful study of such an arrangement in the New England fish industry. 
monopolistic quantity produced by the active seller is high enough to deter entry - this happens, for example, if the distribution of buyers' values is sufficiently convex, and if the marginal cost is not too high.

In Section 5 we compare the equilibrium quantities and prices across three different models (monopoly, competition at one auction site, and two competing auction sites) under the assumption that there is a large number of buyers. We show that, under a mild condition, the monopoly model has the highest price and lowest quantity in equilibrium. Furthermore, if the equilibrium supply is relatively high (e.g., if the production cost is low and the distribution of buyers' valuations is sufficiently convex), the model with two auction sites is more competitive than the model with one auction site: the equilibrium price is lower and the equilibrium quantity is higher in the former model. Finally, if the equilibrium supply is relatively low, then the reverse is true.

Section 6 contains several concluding comments. Appendix A contains several useful results from the literature on order statistics. Appendix B contains all proofs.

\section{The model}

There are two competing sellers and $n \geqslant 2$ buyers. The sellers can each produce several units of a homogenous good. Each buyer has unit demand. The valuation of buyer $j$ for a unit of the good is private information to $j$. Valuations are drawn independently of each other from the interval $[0, T], T \leqslant \infty$, according to a distribution function $F$ that is common knowledge.

We consider two different competition models among the sellers:

1. One auction site: Sellers 1 and 2 simultaneously provide $k_{1}$ and $k_{2}$ units to the auctioneer, respectively. Then the auctioneer sells the $\left(k_{1}+k_{2}\right)$ units via a uniform-price auction where all winners pay the highest losing bid. The revenue of each seller is the product of the equilibrium price and his supplied quantity.

2. Two competing auction sites: In the first stage, sellers 1 and 2 simultaneously choose the number of objects for sale $k_{i}, i=1,2$, in their own, separate auctions. In the second stage, before they learn their valuations, but after they know the decision made by the sellers in the first stage, buyers choose whether to attend auction 1 or 2 . In the third and last stage, each buyer learns his valuation and submits a bid in his selected auction (each of the two auctions is a uniformprice auction as above). The revenue of each seller is the product of his supplied quantity and equilibrium price in his own auction.

\subsection{Notation}

We use the following notation: $X_{k, n}$ denotes $k$ th order statistic out of $n$ independent valuations distributed according to $F$ (note that $X_{n, n}$ is the highest order statistic, and so on). The distribution $F_{k, n}$ and the density $f_{k, n}$ of $X_{k, n}$ are given by

$$
\begin{aligned}
& F_{k, n}(x)=\sum_{j=k}^{n}\left(\begin{array}{l}
n \\
j
\end{array}\right) F(x)^{j}[1-F(x)]^{n-j}, \\
& f_{k, n}(x)=\frac{n !}{(k-1) !(n-k) !} F(x)^{k-1}[1-F(x)]^{n-k} f(x) .
\end{aligned}
$$

We denote by $E X_{k, n}$ the expected value of $X_{k, n}$. 
The failure rate (or hazard rate) of a distribution $F$ is defined by

$$
\lambda(x)=\frac{f(x)}{1-F(x)} .
$$

A distribution function $F$ has an IFR if its failure (or hazard) rate, $\lambda(x)$, is increasing. Analogously, $F$ has an decreasing failure rate (DFR) if $\lambda(x)$ is decreasing. Note that convexity of $F$ implies IFR, while DFR implies concavity. The only distribution that is both concave and convex is the uniform, while the only distribution that is both IFR and DFR is the exponential.

A distribution function $F$ is star-shaped on $[0, T]$ if $F(x) / x$ is increasing in $x$. ${ }^{11}$ Let $X(Z)$ have distributions $F(G)$ such that $F(0)=G(0)=0$. Distribution $F$ is star-shaped with respect to $G$ if the function $G^{-1} F(x)$ is star-shaped. Note that:

1. If $F(0)=0=G(0), G^{-1} F$ convex implies $G^{-1} F$ star-shaped.

2. If $G$ is the exponential distribution, then $G^{-1} F$ convex is equivalent to $F$ being IFR.

3. If $G$ is uniform, then $G^{-1} F$ convex is equivalent to $F$ is convex.

\section{One auction site}

We discuss first the model of a uniform-price multi-object auction with $n$ buyers and a single, monopolist seller with variable supply. The derived insights will be repeatedly used in the analysis of competition among sellers below.

\subsection{Monopoly seller}

The seller decides on the supply $k$, and then the buyers decide what to bid in an uniform-price auction for $k$ units. We do not explicitly analyze here the use of exclusion tools such as reserve prices and entry fees. Thus all $n$ buyers have an incentive to participate in the auction. Note that in an auction with endogenous quantity and a large number of buyers, the marginal gain from setting optimal reserve price is small if quantity is optimally set. The intuition is provided by the fact that the realizations of $X_{n-k . n}$ are very close to $E X_{n-k, n}$ if $n$ is large. The seller can control $E X_{n-k, n}$ by varying the quantity $k$. If the reserve price $\rho$ is binding and enhancing revenue, the seller can always set the quantity to $k^{\prime}$ such that, approximately, $E X_{n-k^{\prime}, n}=\rho$. After this adjustment, the reserve price is redundant.

We assume first that the seller has a zero cost of producing the object. If the seller offers $k>n$ objects, it is obvious that the equilibrium price and profit are equal to zero. Thus, without loss of generality, we restrict attention to the case $k \leqslant n$. In the auction, each of the $k$ highest buyers wins a single unit, and pays the equilibrium price $X_{n-k, n}$. In expectation, the seller's maximization problem is given by

$$
\max _{k} R(k, n)=\max _{k} k E X_{n-k, n} .
$$

The relation between the optimal number of objects and the distribution of the buyers' valuation is as follows:

\footnotetext{
11 The terminology is somewhat obscure, but we keep it here since it is extensively used in the relevant statistical literature.
} 
Proposition 1. Consider two distributions $G, F$ leading to optimal supplies $k_{G}^{*}(n)$ and $k_{F}^{*}(n)$, respectively, when there are $n$ buyers. If $G^{-1} F$ is star-shaped then $k_{G}^{*}(n) \leqslant k_{F}^{*}(n)$.

Proposition 1 implies the following two corollaries:

Corollary 1. If $F$ is IFR $(D F R)$, and $n$ is large, then $k_{F}^{*}(n)$, the optimal supply in an auction with $n$ buyers satisfies $k_{F}^{*}(n) \geqslant(\leqslant) \frac{n}{e}$, where $e$ is the natural logarithm basis.

Corollary 2. If $F$ is convex (concave), then $k_{F}^{*}(n)$, the optimal supply in the auction with $n$ buyers satisfies $k_{F}^{*}(n) \geqslant(\leqslant) \frac{n}{2}$.

The following result considers the function $k_{F}^{*}(n)$ relating the optimal supply to the number of buyers, and it shows that the "slope" of this function is less than unity if $F$ is IFR (thus, the difference $n-k^{*}(n)$ weakly increases in $\left.n\right)$ :

Proposition 2. Let $k_{F}^{*}(n)$ be the optimal number of objects in the auction with $n$ buyers. If $F$ is IFR, then, for all $n, k_{F}^{*}(n+1)-k_{F}^{*}(n) \leqslant 1$.

Remark 1. When $n$ is large, we have the approximation $E X_{n-k, n} \approx F^{-1}\left(\frac{n-k}{n}\right)$. Hence, the seller's revenue is

$$
R_{1}(k, n)=k E X_{n-k, n} \approx k F^{-1}\left(\frac{n-k}{n}\right)=k F^{-1}\left(1-\frac{k}{n}\right) .
$$

Consider now a monopolist seller facing $n$ buyers, each with unit demand and valuation distribution $F$. The monopolist chooses $p$ to maximize his expected revenue.

$$
R_{2}(p, n)=n p[1-F(p)] \text {. }
$$

Setting $k=n[1-F(p)]$, or equivalently $p=F^{-1}\left[1-\frac{k}{n}\right]$, we see that the monopoly and auction models are equivalent if the number of buyers is sufficiently large. In particular, $p^{*}$, the limit equilibrium price in a single auction with endogenous quantity and a sufficiently large number of buyers is determined by the hazard rate:

$$
\lambda\left(p^{*}\right)=\frac{f\left(p^{*}\right)}{1-F\left(p^{*}\right)}=\frac{1}{p^{*}} .
$$

Note also that an increase of the distribution of the buyers' valuations in the hazard rate order (which implies first-order stochastic dominance) yields a higher equilibrium price.

Example 1. Let $F(x)=x^{1 / a}(a>0)$ with support $[0,1]$. When $n$ is large, the seller's revenue if he sells $k$ objects is

$$
R(k, n, a)=k E X_{n-k, n} \approx k F^{-1}\left(\frac{n-k}{n}\right)=k\left(\frac{n-k}{n}\right)^{a} .
$$

The optimal $k$ is $k_{F}^{*}(n)=\frac{1}{a+1} n$ and we have $k_{F}^{*}(n, a)^{*}<n / 2$ if $a>1$ and $k_{F}^{*}(n, a)>n / 2$ if $a<1$. The limit equilibrium price is $p^{*}(a)=\left(\frac{a}{a+1}\right)^{a}$. 
Assume now that the seller faces a constant marginal cost of producing one unit, and denote this cost by $c \geqslant 0$. The seller's maximization problem is

$$
\max _{k} \pi(k, n)=\max _{k} R(k, n)-c k=\max _{k} k\left(E X_{n-k, n}-c\right) .
$$

The marginal revenue from an additional object is given by $R(k+1, n)-R(k, n)$. The following result follows from the observation that the marginal revenue is decreasing in the number of objects if $F$ is IFR.

Proposition 3. If $F$ is IFR, the optimal number of units $k_{F}^{*}(n, c)$ decreases in $c$ for fixed $n$.

\subsection{Competition among sellers at a unique auction site}

We consider now two sellers competing to sell units to $n$ buyers through a single, uniformprice auction. Sellers 1 and 2 provide $k_{1}$ and $k_{2}$ objects to the auctioneer, respectively. Then the auctioneer sells the $\left(k_{1}+k_{2}\right)$ units through the uniform-price auction. Throughout of this section, we assume for simplicity that $c=0$. All main results can be immediately extended to the case of positive marginal costs. Moreover, the main result, existence of equilibria in pure strategies immediately generalizes to a setting with several competing sellers.

If $\left(k_{1}^{*}, k_{2}^{*}\right)$ is an equilibrium, then for all $s \in\left\{-k_{i}^{*}, \ldots, n-k_{i}^{*}-k_{j}^{*}\right\}$ it must hold

$$
k_{i}^{*} E X_{n-k_{i}^{*}-k_{j}^{*}, n}-\left(k_{i}^{*}+s\right) E X_{n-k_{i}^{*}-k_{j}^{*}-s, n} \geqslant 0, \quad i=1,2 .
$$

The following example illustrates several interesting phenomena: (1) symmetric and asymmetric pure-strategy equilibria may coexist; (2) symmetric pure-strategy equilibria may not exist ${ }^{12}$; (3) as the number of buyers gets large, all equilibria converge to the symmetric one.

Example 2. Suppose that $F$ is uniform. Given $k_{2}$, firm 1 chooses $k_{1}$ to maximize

$$
R_{1}=k_{1} E X_{n-\left(k_{1}+k_{2}\right), n}=\frac{k_{1}\left(n-k_{1}-k_{2}\right)}{n+1} .
$$

If $k_{1}$ is optimal, then for any $s=-k_{1}, \ldots, n-k_{1}-k_{2}$

$$
\begin{aligned}
k_{1}\left(n-k_{1}-k_{2}\right)-\left(k_{1}+s\right)\left(n-k_{1}-k_{2}-s\right) & \geqslant 0, \\
s\left(s-n+2 k_{1}+k_{2}\right) & \geqslant 0 .
\end{aligned}
$$

Setting $s=1,-1$, we get

$$
\frac{1}{2}\left(n-k_{2}-1\right) \leqslant k_{1} \leqslant \frac{1}{2}\left(n-k_{2}+1\right) .
$$

Similarly, if $k_{2}$ is optimal for firm 2, we obtain

$$
\frac{1}{2}\left(n-k_{1}-1\right) \leqslant k_{2} \leqslant \frac{1}{2}\left(n-k_{1}+1\right) .
$$

\footnotetext{
12 Amir and Lambson [1] exhibit a sufficient condition for the existence of a unique equilibrium in the standard model of Cournot competition.
} 
Combining Eqs. (2) and (3), we get

$$
\begin{aligned}
& k_{1} \geqslant \frac{1}{2}\left(n-k_{2}-1\right) \geqslant \frac{1}{2}\left(n-\frac{1}{2}\left(n-k_{1}+1\right)-1\right) \Rightarrow k_{1} \geqslant \frac{1}{3}(n-3), \\
& k_{1} \leqslant \frac{1}{2}\left(n-k_{2}+1\right) \leqslant \frac{1}{2}\left(n-\frac{1}{2}\left(n-k_{1}-1\right)+1\right) \Rightarrow k_{1} \leqslant \frac{1}{3}(n+3) .
\end{aligned}
$$

Therefore,

$$
\frac{n}{3}-1 \leqslant k_{1} \leqslant \frac{n}{3}+1 \quad \text { and } \quad \frac{n}{3}-1 \leqslant k_{2} \leqslant \frac{n}{3}+1 .
$$

(1) If $n=3 m$ there are three possible pure-strategy equilibria (see details in the Appendix)

$$
\begin{array}{ll}
k_{1}^{*}=m+1, & k_{2}^{*}=m-1, \\
k_{1}^{*}=m-1, & k_{2}^{*}=m+1, \\
k_{1}^{*}=k_{2}^{*}=m . &
\end{array}
$$

(2) If $n=3 m+1$, there are also three pure-strategy equilibria

$$
\begin{aligned}
& k_{1}^{*}=m+1, \quad k_{2}^{*}=m, \\
& k_{1}^{*}=m, \quad k_{2}^{*}=m+1, \\
& k_{1}^{*}=k_{2}^{*}=m .
\end{aligned}
$$

(3) If $n=3 m+2$, there are only two asymmetric pure-strategy equilibria

$$
\begin{aligned}
& k_{1}^{*}=m, \quad k_{2}^{*}=m+1, \\
& k_{1}^{*}=m+1, \quad k_{2}^{*}=m .
\end{aligned}
$$

We turn now to a proof of existence of pure-strategy Nash equilibria. Since the strategy spaces are discrete and since the payoff functions are not continuous, it is obvious that the standard approach cannot work. Fortunately, it turns out that our game is an ordinal potential game in the sense of Monderer and Shapley [30]. Thus it possesses a Nash equilibrium in pure strategies, corresponding to a maximizer of the potential function. The next two results hold also for the case where $c>0$.

Theorem 1. A pure-strategy Nash equilibrium always exists in the competition model with one auction site.

By taking a closer look at the potential function, we also get the existence of an "almost" symmetric, pure-strategy Nash equilibrium:

Definition 1. An equilibrium $\left(k_{1}^{*}, k_{2}^{*}\right)$ is quasi-symmetric if $\left|k_{1}^{*}-k_{2}^{*}\right| \leqslant 1$.

Theorem 2. A pure-strategy, quasi-symmetric Nash equilibrium always exists in the competition model with one auction site.

Remark 2. Consider now a large number of buyers. If the sellers supply $k_{1}$ and $k_{2}$ units, respectively, the revenues are approximated by

$$
\pi_{i}=k_{i} E X_{n-\left(k_{i}+k_{j}\right), n} \approx k_{i} F^{-1}\left(\frac{n-k_{i}-k_{j}}{n}\right)=k_{i} F^{-1}\left(1-\frac{k_{i}+k_{j}}{n}\right) .
$$


This is equivalent to a standard Cournot model where the inverse demand function is given by

$$
P\left(k_{1}+k_{2}\right)=F^{-1}\left(1-\frac{k_{1}+k_{2}}{n}\right) .
$$

In particular, under the conditions of Amir and Lambson [1], in the limit as $n$ gets large, there exists a unique symmetric equilibrium.

We conclude this section with the following equilibrium characterization result which generalizes the previous results obtained for the monopoly case:

Proposition 4. (1) Let F be convex (concave). Then, in any equilibrium, the total supplied quantity is higher (lower) than $\frac{2}{3} n$. In particular, if the distribution of buyers' valuations is uniform, then the total supplied quantity is $\frac{2 n}{3}$ units. (2) If $F$ is IFR (DFR), and if $n$ is large, then in any equilibrium, the total supplied quantity is higher (lower) than $\frac{2}{1+e} n$. In particular, if the distribution of buyers' values is exponential, the supplied quantity is $\frac{2}{1+e} n$ units.

\section{Competition among auction sites}

We now consider two competing, uniform-price, multi-unit auctions. First, the two sellers simultaneously choose the number of units for sale $k_{i}, i=1,2$ in their respective auctions. The buyers observe the respective supply at each site, and then decide where to bid, and what bid to place. Thus, a buyer's strategy is a function of both observed quantities. Because each auction is uniform, the bidding part of any buyer's decision is simple, and of no further concern: each bidder places a bid equal to his valuation at the respective auction.

Let $\sigma_{j}\left(k_{1}, k_{2}\right)$ denote a deterministic location decision of buyer $j, j=1, \ldots, n$ given observed quantities $k_{1}$ and $k_{2}$. Thus $\sigma_{j}\left(k_{1}, k_{2}\right): N \times N \rightarrow\{1,2\}$.

Definition 2. For any profile of strategies $\left\{k_{1}, k_{2}, \sigma_{1}, \ldots, \sigma_{n}\right\}$ define

$$
n_{i}=n_{i}\left(k_{1}, k_{2}, \sigma_{1}, \ldots, \sigma_{n}\right)=\#\left\{j \mid \sigma_{j}\left(k_{1}, k_{2}\right)=i\right\}, \quad i=1,2 .
$$

That is, $n_{i}$ is the number of buyers attending auction $i$ given the strategy profile.

A profile of strategies $\left\{k_{1}^{*}, k_{2}^{*}, \sigma_{1}^{*}\left(k_{1}, k_{2}\right), \ldots, \sigma_{n}^{*}\left(k_{1}, k_{2}\right)\right\}$ is an equilibrium of the competing auctions model if:

1. For any $k_{1}, k_{2}$, and for any buyer $j, \sigma_{j}^{*}\left(k_{1}, k_{2}\right)$ is a best reply to $\sigma_{-j}^{*}\left(k_{1}, k_{2}\right)$. That is, given quantities chosen by sellers, and given the location decisions of other buyers who have observed these quantities, no buyer $j$ has an incentive to switch to another auction site.

2. For $i=1,2, k_{i}^{*} \in \arg \max _{k_{i}} k_{i} E X_{n_{i}^{*}}\left(k_{i}\right)-k_{i}, n_{i}^{*}\left(k_{i}\right)$ where $n_{i}^{*}\left(k_{i}\right)=n_{i}\left(k_{i}, k_{3-i}^{*}, \sigma_{1}^{*}\left(k_{i}, k_{3-i}^{*}\right)\right.$, $\left.\ldots, \sigma_{n}^{*}\left(k_{i}, k_{3-i}^{*}\right)\right)$. That is, $k_{i}^{*}$ maximizes seller $i$ 's revenue given the quantity decision of the other seller, and given the buyers' location decisions.

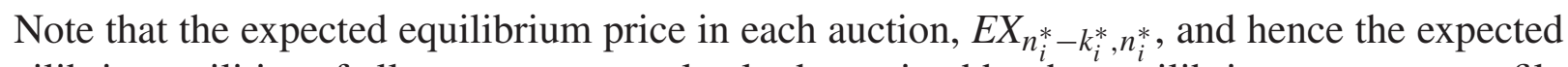
equilibrium utilities of all agents are completely determined by the equilibrium outcome profile $\left(n_{1}^{*}, k_{1}^{*} ; n_{2}^{*}, k_{2}^{*}\right)$. Note also that in an equilibrium with positive seller profits at the two auction sites we cannot have $k_{1}^{*}+k_{2}^{*}=n$. In that case, the supply at one auction site (at least) is no less than the number of buyers, and the respective price must be zero. 
We focus below on equilibrium outcome profiles $\left(n_{1}^{*}, k_{1}^{*} ; n_{2}^{*}, k_{2}^{*}\right)$. The following lemma gives a condition on the two auction prices that must hold in any equilibrium. This condition is useful since it makes operational condition 1 of the above equilibrium definition.

Lemma 1. Consider any fixed strategy profile $\left\{k_{1}, k_{2}, \sigma_{-j}\right\}$ for the two sellers, and for all buyers except buyer $j$. Then, it is a best reply for $j$ to choose the auction with the lower expected price. Moreover, in any equilibrium outcome $\left(n_{1}^{*}, k_{1}^{*} ; n_{2}^{*}, k_{2}^{*}\right)$ it must hold that

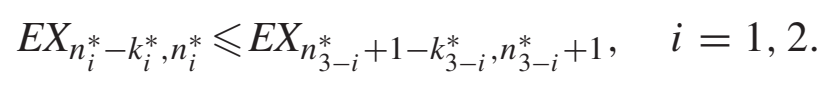

The lemma says that a buyer who contemplates a switch from auction 1 to auction 2, say, must expect at auction 2 a price which is higher than the one at auction 1. Thus, while equilibrium prices at the two auction sites need not be strictly equal, any arbitrage opportunity should vanish if someone tries to actually exploit it. It is important to note that, in our analysis, the computation of expected prices does take into account $j$ 's own effect on price after a switch!

\subsection{Equilibrium coexistence of two auction sites}

Our main result in this section is that two auction sites yielding positive profits for the respective sellers cannot coexist in equilibrium if the distribution of buyers' values is IFR, and if the marginal production cost, $c$, is sufficiently low. The proof is based on the following lemma which has some independent interest. It shows that, for any profile of buyers' actions, at least one of the sellers has an incentive to increase supply if $F$ is IFR.

Lemma 2. (1) Consider any outcome profile $\left(n_{1}, k_{1} ; n_{2}, k_{2}\right)$ where $n_{1}, n_{2}>0, k_{1}, k_{2}>0$ and $k_{1}+k_{2}<n$, and assume that buyers play a best response to any announcement of quantities. If $E X_{n_{i}-k_{i}, n_{i}} \leqslant E X_{n_{j}-k_{j}, n_{j}}, i, j=1,2$, then seller $i$ can attract at least one more buyer by supplying one additional unit.

(2) Assume that $F$ is IFR, and that $c=0$. For any profile $\left(n_{1}, k_{1} ; n_{2}, k_{2}\right)$ where $n_{1}, n_{2}>0$, $k_{1}, k_{2}>0$ and $k_{1}+k_{2}<n$, one of the sellers can increase his profit by supplying one additional unit.

(3) Let $\Delta R(k, n)=R(k+1, n+1)-R(k, n)$ denote the change in revenue of a seller whose supply increases from $k$ to $k+1$ while the number of buyers attending his auction increases from $n$ to $n+1$. If $F$ is IFR, and if $c=0$, then $\Delta R(k, n)$ decreases in $k$ for a fixed $n$.

Although increased supply tends to drive down an auction's equilibrium price, the above lemma shows that, under IFR, it is profitable for a seller with a lower price to supply one additional unit while attracting one extra bidder. This result resembles the well-known result by Bulow and Klemperer who showed that, with decreasing marginal revenue-a condition which is indeed implied by IFR, a seller endowed with one unit can increase revenue by dropping the reserve price if she attracts at least one more bidder [9].

The above lemma yields then:

Theorem 3. Assume that $F$ is IFR. Then, for sufficiently small marginal production costs $c$, there is no equilibrium in which both sellers are active and make positive profits. In particular, if

$$
c<\frac{n}{2} E X_{1, \frac{n}{2}+1}-\left(\frac{n}{2}-1\right) E X_{1, \frac{n}{2}}
$$

there is no symmetric equilibrium with positive profits. 
Note that the right-hand side of inequality (5) can be shown to be positive if $F$ is IFR, so the above condition is well defined. If the IFR condition is not satisfied, or if the cost is high enough (in contrast to the assumptions of the above proposition) an equilibrium where two auction sites are active, and where both sellers make positive profits may then exist. We look first at an example where $F$ does not satisfy the IFR condition:

Example 3. Let $F(x)=x^{1 / 2}$, and let $c=0$. Then

$$
\begin{aligned}
\Delta R\left(k_{i}, n_{i}\right) & =\frac{\left(k_{i}+1\right)\left(n_{i}-k_{i}+1\right)\left(n_{i}-k_{i}\right)}{\left(n_{i}+2\right)\left(n_{i}+3\right)}-\frac{k_{i}\left(n_{i}-k_{i}+1\right)\left(n_{i}-k_{i}\right)}{\left(n_{i}+1\right)\left(n_{i}+2\right)} \\
& =\frac{\left(n_{i}-k_{i}\right)\left(n_{i}-k_{i}+1\right)\left(n_{i}+1-2 k_{i}\right)}{\left(n_{i}+1\right)\left(n_{i}+2\right)\left(n_{i}+3\right)} .
\end{aligned}
$$

Note that $\Delta R\left(k_{i}, n_{i}\right) \leqslant 0$ if $k_{i} \geqslant \frac{n_{i}+1}{2}$. Letting $k_{1}=k_{2}=2$ and $n_{1}=n_{2}=3$, it is clear that no seller has an incentive to increase supply. Assume then that seller 1, say, lowers supply to one unit. Then a buyer attending his auction has an incentive to switch to the other auction since there the price will be $E X_{2,4}<E X_{2,3}$. Therefore no seller has an incentive to deviate, and we obtain a symmetric equilibrium in which every seller sells two units and makes a positive profit. ${ }^{13}$

Next we look at situations where the marginal cost $c$ is sufficiently high. Then, sellers do not want to increase their supply above a certain level (since the price drops below cost), and an equilibrium may exist.

If a seller sells $k^{*}$ units in a symmetric equilibrium, he should have no incentive to increase supply to $k^{*}+1$. Thus, for $F$ which is IFR we have the necessary condition

$$
p=E X_{\frac{n}{2}-k^{*}, \frac{n}{2}}>c>\left(k^{*}+1\right) E X_{\frac{n}{2}-k^{*}, \frac{n}{2}+1}-k^{*} E X_{\frac{n}{2}-k^{*}, \frac{n}{2}},
$$

where the left inequality indicates that a seller makes a positive profit by selling $k^{*}$ units to half the number of buyers, and the right inequality indicates that a seller has no incentive to increase the number of supplied units from $k^{*}$ to $k^{*}+1$ if by doing so the number of buyers attending his auction increases from $\frac{n}{2}$ to $\frac{n}{2}+1 .^{14}$

The following proposition provides a sufficient condition for existence of a symmetric equilibrium with the maximal supply value where both sellers make positive profits.

Proposition 5. Assume that $F$ is IFR. If

$$
\left(\frac{n}{2}-1\right) E X_{\left(2, \frac{n}{2}+1\right)}-\left(\frac{n}{2}-2\right) E X_{\left(2, \frac{n}{2}\right)}>c>\frac{n}{2} E X_{\left(1, \frac{n}{2}+1\right)}-\left(\frac{n}{2}-1\right) E X_{\left(1, \frac{n}{2}\right)}
$$

then the profile where each seller sells $\frac{n}{2}-1$ objects to $\frac{n}{2}$ buyers constitutes a symmetric equilibrium outcome.

We close this section by addressing the role of timing assumptions for equilibrium existence/inexistence results. In our model, buyers first observe offered quantities before deciding where to bid. A seller with a lower expected price can always "poach" one bidder away from the other auction site by supplying one more unit. If the distribution of values is IFR, such poaching

\footnotetext{
${ }^{13}$ In this example there is also a symmetric equilibrium where each seller supplies three units, and where profits are zero.

${ }^{14}$ If $F$ is IFR, by Lemma 2 the RHS of (6) is always positive.
} 
increases her profits. In contrast, with buyers and sellers deciding simultaneously, poaching is not profitable because increasing one's supply (while holding fixed the buyers' location choice), would only lower own price and thus profit. The next example shows how two auctions sites of different size can indeed coexist in equilibrium for an IFR distribution and for a zero marginal cost if all agents move simultaneously, as in [16]. This should be contrasted to the non-existence result exhibited in Theorem 3.

Example 4. Assume now that buyers and sellers move simultaneously (i.e., sellers decide how much to supply at their respective sites, and buyers decide which auction to attend without seeing the supplied quantities). Suppose that $\left(n_{1}, k_{1} ; n_{2}, k_{2}\right)$ is an equilibrium outcome. Then the following two conditions must hold: (1) Seller $i$ does not have incentive to change quantity $k_{i} ;$; $)$ No buyer has an incentive to move to the other auction site. Condition 1 implies that $k_{i}$ must be the monopolistic quantity given the number of buyers $n_{i}$ in auction site $i$. In order to prevent a buyer from switching locations, a necessary and sufficient condition is

$$
E X_{n_{i}-k_{i}, n_{i}}<E X_{n_{3-i}+1-k_{3-i}, n_{3-i}+1}, \quad i=1,2
$$

and an analogous conditions must hold if the expected price in auction 1 is the lower one. Now suppose the distribution is uniform, and let $n=2\left(m_{1}+m_{2}\right)$ where $m_{1} \geqslant m_{2}$. Consider

$$
\begin{aligned}
& n_{1}=2 m_{1}, \quad n_{2}=2 m_{2}, \\
& k_{1}=m_{1}, \quad k_{2}=m_{2}
\end{aligned}
$$

so that the sellers do not have incentive to deviate. Note that

$$
E X_{n_{1}-k_{1}, n_{1}}=\frac{m_{1}}{2 m_{1}+1} \geqslant \frac{m_{2}}{2 m_{2}+1}=E X_{n_{2}-k_{2}, n_{2}} .
$$

Thus $\left(2 m_{1}, m_{1} ; 2 m_{2}, m_{2}\right)$ is an equilibrium if

$$
E X_{n_{1}-k_{1}, n_{1}}<E X_{n_{2}+1-k_{2}, n_{2}+1} \Leftrightarrow \frac{m_{1}}{2 m_{1}+1}<\frac{m_{2}+1}{2 m_{2}+1+1}=\frac{1}{2}
$$

which trivially holds. To conclude, we obtain that $\left(2 m_{1}, m_{1} ; 2 m_{2}, m_{2}\right)$ is an equilibrium for any $m_{1}, m_{2}>0$, such that $n=2\left(m_{1}+m_{2}\right)$.

\subsection{A unique auction site as the outcome of competition}

We now focus on equilibria where only one auction site is active, and where only one seller makes a positive profit. Roughly speaking, such an equilibrium may exist if the optimal monopolistic quantity for the unique active seller is high enough. Then, the other seller cannot convince enough buyers to switch to his own auction, and thus prefers to stay out.

Formally, an equilibrium outcome with the form $\left(n_{1}^{*}=n, k_{1}^{*}>0, n_{2}^{*}=0, k_{2}^{*}=0\right)$ exists if and only if:

1. $\quad k_{1}^{*}=\arg \max _{k} k\left(E X_{n-k, n}-c\right)>0$

and

2. for all $k_{2} \leqslant n-k_{1}^{*}$, either the set $M=M\left(k_{2}\right)=\left\{m \mid m \leqslant n-k_{1}^{*} \wedge E X_{m-k_{2}, m} \geqslant c\right\}$ is empty or

$$
E X_{\widetilde{n}_{2}-k_{2}, \widetilde{n}_{2}}>E X_{n-\widetilde{n}_{2}+1-k_{1}^{*}, n-\widetilde{n}_{2}+1},
$$

where $\tilde{n}_{2}=\tilde{n}_{2}\left(k_{2}\right)$ is the minimal element in $M$. 
Condition (8) requires that seller 1 optimizes his (monopolistic) supply and makes a positive profit, given that seller 2 is not active. In condition (9), $\tilde{n}_{2}$ is the minimal number of buyers for which selling $k_{2}$ units is profitable for seller 2 . Note that if condition (9) holds and if $n_{2}>\tilde{n}_{2}$, we also get

$$
E X_{n_{2}-k_{2}, n_{2}}>E X_{\widetilde{n}_{2}-k_{2}, \widetilde{n}_{2}}>E X_{n-\widetilde{n}_{2}+1-k_{1}^{*}, n-\widetilde{n}_{2}+1}=E X_{n-n_{2}+\left(n_{2}-\widetilde{n}_{2}+1\right)-k_{1}^{*}, n-n_{2}+\left(n_{2}-\widetilde{n}_{2}+1\right)}
$$

The first inequality follows by repeated application of the known relation $E X_{(i+1, n+1)} \geqslant E X_{(i, n)}$, and the second inequality is condition (9). That is, condition (9) guarantees that if $n_{2}>\tilde{n}_{2}$ buyers attend auction 2 , at least $\left(n_{2}-\tilde{n}_{2}+1\right)$ buyers will want to switch to auction 1 . Thus, under condition (9), seller 2 must remain inactive, and makes zero profit.

Proposition 6. (1) Assume that $k_{1}^{*}=\arg \max _{k} k\left(E X_{n-k, n}-c\right)$ is sufficiently large. (For example, assume that $F$ is sufficiently convex and that $c$ is sufficiently small.) Then there exists an asymmetric equilibrium with a unique active seller.

(2) Assume that $k_{1}^{*}=\arg \max _{k} k\left(E X_{n-k, n}-c\right)<\frac{n}{2}$. (For example, assume that $F$ is sufficiently concave.) Then there exists no equilibrium with a unique active seller.

The following example illustrates the result.

Example 5. Let $c=0, n=4$ and let $F$ be uniform on $[0,1]$. By Corollary 2 the optimal supply for a monopolistic seller 1 is $k_{1}^{*}=2$. The only alternative for seller 2 that leads to a positive profit is to supply one unit. Since $E X_{1,2}-E X_{4-1-2,4-1}=E X_{1,2}-E X_{1,3}>0$, condition (9) is satisfied. Seller 2 cannot attract more than one buyer to his auction, and he therefore prefers to stay out.

\section{Comparison among models}

How do the models analyzed in this paper (in particular the competition models with either one or two auctions sites) compare in terms of equilibrium prices and quantities? In this section we answer this question under the assumption that the number of buyers is large. Quite interestingly, the answer is not clear cut. We consider the following:

- Monopoly (M): one auction site with one seller and $n$ buyers.

- Competition among sellers at one auction site (C1): two competing sellers at one auction site with $n$ buyers.

- Competing auctions (C2): two sellers running separate auctions, competing to attract $n$ buyers. For the results below we need of course to assume that a symmetric equilibrium exists in these models (recall that this is not generally true for model C2).

Proposition 7. Assume that $F$, the distribution of buyers' values, is IFR. Assume that total equilibrium supply in all three models is at least $\frac{n}{3}+2$. (This happens, for example, if either $F$ is convex enough with respect to the exponential distribution, or if the marginal cost is not too high.) Then the following hold:

(1) The equilibrium price under monopoly is higher than the prices in the symmetric equilibria of the competition models.

(2) If total supply is higher (lower) than $\frac{n}{2}+2$, then the equilibrium price in the competition model with one auction site is higher (lower) than the equilibrium price in the competition model with two auction sites. 


\section{Concluding remarks}

We have studied competition among two sellers who optimize their respective supply in markets with a finite, not necessarily large, number of buyers. We examined a model where all transactions take place at price determined in one auction, and another where two separate auction sites may operate side by side. A unified perspective on both models can be obtained by considering buyers' switching costs. Whereas an equilibrium where both sellers make profits always exists in the first model (one auction site), this is not the case in the second model (two auction sites) under ubiquitous conditions on the demand function (decreasing marginal revenue) and on the supply functions (sufficiently low marginal costs). In markets with sinking production or trading costs, our results suggest a movement towards dominant market places featuring several competing suppliers. Ebay is an obvious example.

Instead of an earlier competition mode characterized by poaching business from competitors (which corresponds to the insight obtained from our model with competing auction sites), we currently observe a large move towards consolidation in the trading of securities. ${ }^{15}$ For example, on October 17th 2006 the Chicago Mercantile Exchange agreed to buy the Chicago Board of Trade, thus aiming to create the world's biggest financial marketplace. ${ }^{16}$ Similarly, the Deutsche Börse has long argued for the need of a pan-European exchange, and tried (unsuccessfully) to buy the London Stock Exchange-which is now on NASDAQ's buying list. ${ }^{17}$

Last but not least, we hope that the mathematical methods used in this paper will prove to be useful in a variety of other competition models with a small number of traders.

\section{Acknowledgments}

We wish to thank Stefan Behringer, Drew Fudenberg, Phil Haile, two anonymous referees and an associate editor for very helpful comments. We are grateful for the financial support from the German Science Foundation (SFB TR-15), the Max Planck Society, and the Humboldt Foundation.

\section{Appendix A}

Barlow and Porschan [6] proved the following five results about order statistics:

Lemma 3. Assume that $F(0)=0$. If $F$ is convex (concave) then $(n+1) E X_{i, n} / i$ is decreasing (increasing) in $i$ and increasing (decreasing) in $n$.

Lemma 4. Let $G^{-1} F$ be star-shaped on the support of $F$ and assume $F(0)=G(0)=0$. Then $E X_{i n} / E Y_{\text {in }}$ is decreasing in $i$.

\footnotetext{
15 See "The Big Squeeze", The Economist, October 7th, 2006, p. 83.

16 See "Chicago Bulls", in The Economist, October 21th, 2006. p. 88. In another recent instance three North European exchanges unified their listings.

${ }^{17}$ Matros and Zapechelnyuk characterize the revenue maximizing mechanism of a monopolistic mediator between a seller endowed with one unit and several buyers. It would be very interesting to extend their analysis by allowing several sellers and/or several mediators [26].
} 
Lemma 5. If $F$ is $\operatorname{IFR}(D F R)$ and if $F(0)=0$, then $\sum_{1}^{r}(n-i+1)\left(X_{i, n}-X_{i-1, n}\right)$ is stochastically increasing (decreasing) in $n \geqslant r$.

Lemma 6. If $F$ is IFR $(D F R)$ and if $F(0)=0$, then $(n-i+1)\left(X_{i, n}-X_{i-1, n}\right)$ is stochastically increasing (decreasing) in $n \geqslant i$ for fixed $i$.

Lemma 7. If $F$ is IFR $(D F R)$ and if $F(0)=0$, then $(n-i+1)\left(X_{i, n}-X_{i-1, n}\right)$ is stochastically decreasing (increasing) in $i \leqslant n$ for fixed $n$.

\section{Appendix B}

Proof of Proposition 1. If the distribution of buyers' valuations is $F$, the seller's revenue when he sells $k$ units is

$$
k E X_{n-k, n}=(n-r) E X_{r, n} \text { where } r \equiv n-k .
$$

Suppose that $r^{*}=n-k_{F}^{*}(n)$ is optimal: selling $k_{F}^{*}(n)$ is always better than selling $k^{\prime}>k_{F}^{*}(n)$. Letting $r^{\prime}=n-k^{\prime}<r^{*}$, we obtain

$$
\left(n-r^{*}\right) E X_{r^{*}, n} \geqslant\left(n-r^{\prime}\right) E X_{r^{\prime}, n} \Leftrightarrow \frac{E X_{r^{*}, n}}{E X_{r^{\prime}, n}} \geqslant \frac{n-r^{\prime}}{n-r^{*}} .
$$

For distribution $G$, the seller's revenue when he sells $k$ units is

$$
(n-k) E Y_{n-k, n}=(n-r) E Y_{r, n} \quad \text { where } r \equiv n-k .
$$

Suppose that $k_{G}^{*}(n)$ units are now optimal. In order to show that $k_{G}^{*}(n) \leqslant k_{F}^{*}(n)$, it is sufficient to show that under distribution $G$, the seller's revenue of selling $k_{F}^{*}(n)$ units is always larger than when selling $k^{\prime}>k_{F}^{*}(n)$. That is, we need to show that, for $r^{*}>r^{\prime}$

$$
\left(n-r^{*}\right) E Y_{r^{*}, n} \geqslant\left(n-r^{\prime}\right) E Y_{r^{\prime}, n} \Leftrightarrow \frac{E Y_{r^{*}, n}}{E Y_{r^{\prime}, n}} \geqslant \frac{n-r^{\prime}}{n-r^{*}} .
$$

Given condition (10), it is sufficient to show that

$$
\frac{E Y_{r^{*}, n}}{E Y_{r^{\prime}, n}} \geqslant \frac{E X_{r^{*}, n}}{E X_{r^{\prime}, n}} \Leftrightarrow \frac{E X_{r^{*}, n}}{E Y_{r^{*}, n}} \leqslant \frac{E X_{r^{\prime}, n}}{E Y_{r^{\prime}, n}} .
$$

By Lemma 4 in Appendix A, $E X_{i n} / E Y_{i n}$ is decreasing in $i$. Since $r^{*}>r^{\prime}$, the last inequality must hold.

Proof of Corollary 1. When $n$ is large, the optimal number of objects $k_{G}^{*}(n)$ for the exponential distribution $G$ is

$$
\begin{aligned}
k_{G}^{*}(n) \in \arg \max _{k} k E X_{n-k, n} & \approx \arg \max _{k} k G^{-1}\left[\frac{n-k}{n}\right] \\
& =\arg \max \left(k \ln \frac{n}{k}\right) \Rightarrow k_{G}^{*}(n) \approx \frac{n}{e} .
\end{aligned}
$$

$F(0)=G(0)=0$ and $G^{-1} F$ convex imply together that $G^{-1} F$ star-shaped. If $G$ is the exponential distribution, $G^{-1} F$ convex (where finite) is equivalent to $F$ being IFR. Thus, applying 
Proposition 1, we obtain that $k_{F}^{*}(n) \geqslant n / e$ if $F$ is IFR. The reverse inequality holds for $F$ which is DFR.

Proof of Corollary 2. If $G$ is uniform distribution, the optimal number of objects $k_{G}^{*}(n)$ is

$$
k_{G}^{*}(n) \in \arg \max k E X_{n-k, n}=\arg \max \frac{k(n-k)}{n+1} \Rightarrow k_{G}^{*}(n)=\frac{n}{2} .
$$

Applying Proposition 1 , we obtain that $k_{F}^{*}(n) \geqslant(\leqslant) \frac{n}{2}$ if $F$ is convex (concave).

Proof of Proposition 2. By Lemma 6 in Appendix A, if $F$ is IFR (DFR), then $(n-i+1)\left(E X_{i, n}-\right.$ $E X_{i-1, n}$ ) is stochastically increasing (decreasing) in $n \geqslant i$ for fixed $i$. Thus, if $F$ is IFR we obtain

$$
(n-i+2)\left(E X_{i, n+1}-E X_{i-1, n+1}\right)>(n-i+1)\left(E X_{i, n}-E X_{i-1, n}\right) .
$$

This implies that

$$
\begin{aligned}
& (n-i+1) E X_{i, n+1}-(n-i+2) E X_{i-1, n+1}+E X_{i, n+1} \\
& \quad>(n-i) E X_{i, n}-(n-i+1) E X_{i-1, n}+E X_{i, n} .
\end{aligned}
$$

Since $E X_{i, n+1}<E X_{i, n}$ we obtain

$$
(n-i+1) E X_{i, n+1}-(n-i+2) E X_{i-1, n+1}>(n-i) E X_{i, n}-(n-i+1) E X_{i-1, n} .
$$

Letting $i=n-k$ in the above inequality yields

$$
(k+1) E X_{n+1-(k+1), n+1}-(k+2) E X_{n+1-(k+2), n+1}>k E X_{n-k+, n}-(k+1) E X_{n-(k+1), n} .
$$

Thus, if it is profitable to decrease the number of objects from $k+1$ to $k$ when the number of buyers is $n$, then it is also profitable to decrease the number of objects from $k+2$ to $k+1$ when the number of buyers is $n+1$. In particular, $k_{F}^{*}(n+1) \leqslant k_{F}^{*}(n)+1$.

Proof of Proposition 3. By Lemma 7 in Appendix A, if $F$ is IFR, then $(n-i+1)\left(E X_{i, n}-E X_{i-1, n}\right)$ is stochastically decreasing in $i \leqslant n$ for fixed $n$. Thus, if $F$ is IFR we obtain

$$
(n-i+1)\left(E X_{i, n}-E X_{i-1, n}\right)>(n-(i+1)+1)\left(E X_{i+1, n}-E X_{i, n}\right) .
$$

Letting $k=n-i$ in the last inequality yields

$$
\begin{aligned}
& k E X_{n-k, n}-(k+1) E X_{n-(k+1), n}+E X_{n-k, n} \\
& \quad>(k-1) E X_{n-(k-1), n}-k E X_{n-k, n}+E X_{n-(k-1), n} .
\end{aligned}
$$

Since $E X_{n-k, n}<E X_{n-(k-1), n}$ we obtain

$$
k E X_{n-k, n}-(k-1) E X_{n-(k-1), n}>(k+1) E X_{n-(k+1), n}-k E X_{n-k, n} .
$$

The last inequality indicates that the marginal revenue of a seller is decreasing in the number of objects, and this property implies the desired result.

Derivations for Example 2. (1) If $n=3 m$, where $m$ is an integer, inequality 4 becomes

$$
m-1 \leqslant k_{1} \leqslant m+1 \Rightarrow k_{1}=m-1, m \text { or } m+1 \text {. }
$$


Suppose $k_{1}^{*}=m-1$, then

$$
\pi_{2}=\frac{k_{2}\left(n-k_{1}-k_{2}\right)}{n+1}=\frac{k_{2}\left(2 m+1-k_{2}\right)}{3 m+1} \Rightarrow k_{2}^{*}=m \quad \text { or } \quad k_{2}^{*}=m+1 .
$$

But if $k_{2}^{*}=m$, the best response of seller 1 is $k_{1}=m$. Therefore, $k_{1}^{*}=m-1$ and $k_{2}^{*}=m$ is not an equilibrium profile. If $k_{2}^{*}=m+1$, the best response for firm 1 is $k_{1}=m$ or $m-1$. Thus, there are three possible pure-strategy equilibria

$$
\begin{aligned}
& k_{1}=m+1, \quad k_{2}=m-1, \\
& k_{1}=m-1, \quad k_{2}=m+1, \\
& k_{1}=k_{2}=m .
\end{aligned}
$$

(2) If $n=3 m+1$, where $m$ is an integer, inequality 4 becomes

$$
m-\frac{2}{3} \leqslant k_{1} \leqslant m+\frac{4}{3} \Rightarrow k_{1}=m \text { or } m+1 \text {. }
$$

Suppose $k_{1}^{*}=m$, then

$$
\begin{aligned}
\pi_{2} & =\frac{k_{2}\left(n-k_{1}-k_{2}\right)}{n+1}=\frac{k_{2}\left(3 m+1-m-k_{2}\right)}{n+1}=\frac{k_{2}\left(2 m+1-k_{2}\right)}{3 m+2} \\
& \Rightarrow k_{2}^{*}=m \quad \text { or } \quad m+1 .
\end{aligned}
$$

Suppose $k_{1}^{*}=m+1$, then

$$
\pi_{2}=\frac{k_{2}\left(n-k_{1}-k_{2}\right)}{n+1}=\frac{k_{2}\left(3 m+1-m-1-k_{2}\right)}{n+1}=\frac{k_{2}\left(2 m-k_{2}\right)}{3 m+2} \Rightarrow k_{2}^{*}=m .
$$

Again, there will be three pure-strategy equilibria

$$
\begin{aligned}
& k_{1}=m+1, k_{2}=m, \\
& k_{1}=m, k_{2}=m+1, \\
& k_{1}=k_{2}=m .
\end{aligned}
$$

(3) If $n=3 m+2$, where $m$ is an integer, inequality 4 becomes

$$
m-\frac{1}{3} \leqslant k_{1} \leqslant m+\frac{5}{3} \Rightarrow k_{1}=m \text { or } m+1 .
$$

Suppose $k_{1}^{*}=m$, then

$$
\pi_{2}=\frac{k_{2}\left(n-k_{1}-k_{2}\right)}{n+1}=\frac{k_{2}\left(3 m+2-m-k_{2}\right)}{n+1}=\frac{k_{2}\left(2 m+2-k_{2}\right)}{3 m+2} \Rightarrow k_{2}^{*}=m+1 .
$$

Therefore, there will be two asymmetric pure-strategy equilibria

$$
\begin{aligned}
& k_{1}=m, \quad k_{2}=m+1, \\
& k_{1}=m+1, \quad k_{2}=m .
\end{aligned}
$$

Proof of Theorem 1. Since profits are zero if the produced quantity is larger than the number of bidders, we can assume without loss of generality that the sellers choose quantities out of a finite 
set of integers: $\Omega=\{0,1,2, \ldots, K\}, K<\infty$. Given $k_{2}$, seller 1 chooses $k_{1} \in \Omega$ to maximize the payoff function

$$
R_{1}\left(k_{1}, k_{2}\right)=k_{1} E X_{n-\left(k_{1}+k_{2}\right), n}
$$

and given $k_{1}$, seller 2 chooses $k_{2} \in \Omega$ to maximize the payoff function

$$
R_{2}\left(k_{1}, k_{2}\right)=k_{2} E X_{n-\left(k_{1}+k_{2}\right), n}
$$

Let $\Gamma$ denote the above described two-person game, and define

$$
P\left(k_{1}, k_{2}\right)=k_{1} k_{2} E X_{n-\left(k_{1}+k_{2}\right), n}
$$

We now verify that $P$ is an ordinal potential of $\Gamma .{ }^{18}$ We need to check that

$$
\begin{aligned}
& \forall k_{2}>0, \quad R_{1}\left(k_{1}, k_{2}\right)-R_{1}\left(k_{1}^{\prime}, k_{2}\right)>0 \Leftrightarrow P\left(k_{1}, k_{2}\right)-P\left(k_{1}^{\prime}, k_{2}\right)>0, \\
& \forall k_{1}>0, \quad R_{2}\left(k_{1}, k_{2}\right)-R_{2}\left(k_{1}, k_{2}^{\prime}\right)>0 \Leftrightarrow P\left(k_{1}, k_{2}\right)-P\left(k_{1}, k_{2}^{\prime}\right)>0 .
\end{aligned}
$$

The above conditions become

$$
\begin{aligned}
& k_{1} E X_{n-\left(k_{1}+k_{2}\right), n}-k_{1}^{\prime} E X_{n-\left(k_{1}^{\prime}+k_{2}\right), n}>0 \Leftrightarrow k_{1} k_{2} E X_{n-\left(k_{1}+k_{2}\right), n}-k_{1}^{\prime} k_{2} E X_{n-\left(k_{1}^{\prime}+k_{2}\right), n}>0, \\
& k_{2} E X_{n-\left(k_{1}+k_{2}\right), n}-k_{2}^{\prime} E X_{n-\left(k_{1}^{\prime}+k_{2}\right), n}>0 \Leftrightarrow k_{1} k_{2} E X_{n-\left(k_{1}+k_{2}\right), n}-k_{1} k_{2}^{\prime} E X_{n-\left(k_{1}^{\prime}+k_{2}\right), n}>0
\end{aligned}
$$

which trivially hold.

Since both $k_{1}$ and $k_{2}$ are chosen from a finite set $\Omega$, it is obvious that the potential $P$ has a maximum on $\Omega \times \Omega$. (Note that the maximum need not be unique.) The existence result obtains now directly from the following result:

Lemma 8 (Monderer and Shapley [30, Lemma 2.1]). Let $P$ be an ordinal potential function for $\Gamma$. Then the equilibrium set of $\Gamma\left(R_{1}, R_{2}\right)$ coincides with the equilibrium set of $\Gamma(P, P)$. That is, $\left(k_{1}, k_{2}\right) \in \Omega \times \Omega$ is an equilibrium point for $\Gamma$ if and only if for every $i \in\{1,2\}$,

$$
P\left(k_{i}, k_{-i}\right) \geqslant P\left(k_{i}^{\prime}, k_{-i}\right) \quad \text { for every } k_{i}^{\prime} \in \Omega \text {. }
$$

Consequently, if $P$ admits a maximal value in $\Omega \times \Omega$, then $\Gamma\left(R_{1}, R_{2}\right)$ possesses a pure-strategy equilibrium.

Proof of Theorem 2. By the above proof, the potential $P$ has a maximum. Suppose that this maximum is achieved at $\left(k_{1}^{*}, k_{2}^{*}\right)$. We need to show that $\left|k_{1}^{*}-k_{2}^{*}\right| \leqslant 1$. Assume, by contradiction, that the opposite holds and assume without loss of generality that

$$
k_{1}^{*} \geqslant k_{2}^{*}+2
$$

Consider now $\left(k_{1}^{\prime}, k_{2}^{\prime}\right)$ in $\Omega \times \Omega$ defined by

$$
k_{1}^{\prime}=k_{1}^{*}-1, \quad k_{2}^{\prime}=k_{2}^{*}+1
$$

\footnotetext{
${ }^{18}$ If the firms face a marginal cost $c>0$, define the potential $P\left(k_{1}, k_{2}\right)=k_{1} k_{2}\left(E X_{n-\left(k_{1}+k_{2}\right), n}-c\right)$. This and the next proof proceed then in an analogous fashion yielding the respective results.
} 
and observe that $k_{1}^{\prime}+k_{2}^{\prime}=k_{1}^{*}+k_{2}^{*}$. We obtain then that

$$
\begin{aligned}
P\left(k_{1}^{\prime}, k_{2}^{\prime}\right)-P\left(k_{1}^{*}, k_{2}^{*}\right) & =\left(k_{1}^{*}-1\right)\left(k_{2}^{*}+1\right) E X_{n-\left(k_{1}^{*}+k_{2}^{*}\right), n}-k_{1}^{*} k_{2}^{*} E X_{n-\left(k_{1}^{*}+k_{2}^{*}\right), n} \\
& =\left(k_{1}^{*}-k_{2}^{*}-1\right) E X_{n-\left(k_{1}^{*}+k_{2}^{*}\right), n}>0,
\end{aligned}
$$

where the last inequality follows by (11). This yields a contradiction to the assumption that $P$ achieves a maximum at $\left(k_{1}^{*}, k_{2}^{*}\right)$. Thus, $\left|k_{1}^{*}-k_{2}^{*}\right| \leqslant 1$ as desired.

Proof of Proposition 4. (1) Suppose $F$ is convex. Given $k_{2}$, seller 1 chooses $k_{1}$ to maximize

$$
R_{1}=k_{1} E X_{\left(n-k_{2}\right)-k_{1}, n}
$$

By Corollary 2, if $F$ is convex, $k_{1} \geqslant \frac{1}{2}\left(n-k_{2}\right)$. Analogously, we have $k_{2} \geqslant \frac{1}{2}\left(n-k_{1}\right)$. Combining the two inequalities, it follows that $k_{1}+k_{2} \geqslant \frac{2}{3} n$. The opposite inequality holds for concave $F$.

(2) Now suppose $F$ is IFR and $n$ is large. Given $k_{2}$, seller 1 chooses $k_{1}$ to maximize

$$
R_{1}=k_{1} E X_{\left(n-k_{2}\right)-k_{1}, n}
$$

By Corollary 1 , we have $k_{1} \geqslant \frac{1}{e}\left(n-k_{2}\right)$. Similarly, we have $k_{2} \geqslant \frac{1}{e}\left(n-k_{1}\right)$. Therefore $k_{1}+$ $k_{2} \geqslant \frac{2}{1+e} n$. The proof for the DFR case is analogous.

Proof of Lemma 1. Fix a strategy profile for all agents except buyer $j$, and assume that the resulting quantities and number of bidders at each site are $k_{1}, k_{2}, n_{1}, n_{2}$, where $n_{1}+n_{2}=n-1$. Denote by $X$ the random variable representing bidder $j$ 's valuation. If $j$ joins site $i, i=1,2$, his expected payoff is given by

$$
\operatorname{Pr}\left\{X \geqslant E X_{n_{i}+1-k_{i}, n_{i}+1}\right\} E\left[X-E X_{n_{i}+1-k_{i}, n_{i}+1} \mid X \geqslant E X_{n_{i}+1-k_{i}, n_{i}+1}\right] .
$$

It is immediate that the above expression is higher for $i=\arg \min \left\{E X_{n_{1}+1-k_{1}, n_{1}+1}\right.$, $\left.E X_{n_{2}+1-k_{2}, n_{2}+1}\right\}$, thus it is optimal for bidder $j$ to join the site with the lower expected price. The second claim follows then immediately by the definition of equilibrium.

Proof of Lemma 2. (1) Suppose without loss of generality that $E X_{n_{1}-k_{1}, n_{1}} \leqslant E X_{n_{2}-k_{2}, n_{2}}$. Since

$$
E X_{n_{1}+1-\left(k_{1}+1\right), n_{1}+1}=E X_{n_{1}-k_{1}, n_{1}+1}<E X_{n_{1}-k_{1}, n_{1}}
$$

we obtain

$$
E X_{n_{1}+1-\left(k_{1}+1\right), n_{1}+1}<E X_{n_{2}-k_{2}, n_{2}} .
$$

The last inequality provides a sufficient condition for a buyer to move from auction 2 to auction 1 if seller 1 increases the supplied quantity by one unit while all other agents stay put.

(2) Let $n_{1}=m$. By Lemma 5 in Appendix A, if $F$ is IFR then $\sum_{1}^{r}(m-i+1)\left(E X_{i, m}-E X_{i-1, m}\right)$ is stochastically increasing in $m \geqslant r$. Let $r=1$, then

$$
m E X_{1, m}<(m+1) E X_{1, m+1} .
$$

Since $E X_{1, m+1}<E X_{1, m}$, we have

$$
(m-1) E X_{1, m}<m E X_{1, m+1} \text {. }
$$


Similarly, by Lemma 5

$$
E X_{1, m}+(m-1) E X_{2, m}<E X_{1, m+1}+m E X_{2, m+1} .
$$

Since $E X_{2, m+1}<E X_{2, m}$, we have

$$
(m-2) E X_{2, m}<(m-1) E X_{2, m+1} .
$$

By induction, for all $1 \leqslant i<m$,

$$
(m-i) E X_{i, m}<(m-i+1) E X_{i, m+1} .
$$

Letting $i=m-k$, we get

$$
k E X_{m-k, m}<(k+1) E X_{m-k, m+1} .
$$

The last inequality implies that a seller increases his revenue by attracting one more buyer from the other site.

(3) Suppose that the number of buyers in the auction is $m$. By Lemma 6 , if $F$ is IFR (DFR) then $(m-i+1)\left(E X_{i, m}-E X_{i-1, m}\right)$ is stochastically increasing (decreasing) in $m \geqslant i$ for fixed $i$. Thus,

$$
(m-i+2)\left(E X_{i, m+1}-E X_{i-1, m+1}\right)>(m-i+1)\left(E X_{i, m}-E X_{i-1, m}\right) .
$$

This implies that

$$
\begin{aligned}
& (m-i+2) E X_{i, m+1}-(m-i+1) E X_{i, m} \\
& \quad>(m-i+2) E X_{i-1, m+1}-(m-i+1) E X_{i-1, m} .
\end{aligned}
$$

Since $E X_{i, m+1}<E X_{i, m}$ we obtain

$$
(m-i+1) E X_{i, m+1}-(m-i) E X_{i, m}>(m-i+2) E X_{i-1, m+1}-(m-i+1) E X_{i-1, m} .
$$

Letting $i=m-k$, we get

$$
(k+1) E X_{m-k, m+1}-k E X_{m-k, m}>(k+2) E X_{m-k-1, m+1}-(k+1) E X_{m-k-1, m} .
$$

The last inequality implies that the marginal revenue of a seller that attracts one more buyer by supplying one more unit is decreasing in $k$.

Proof of Theorem 3. As mentioned in the text, if both sellers make a profit in equilibrium, we must have $k_{1}^{*}+k_{2}^{*}<n$. First we can rewrite the inequality (12) as

$$
(k+1) E X_{s-k, s+1}-k E X_{s-k, s}>0 \text { for all } s \in[1, n-1] \text { and } k \leqslant s-1 .
$$

Now assume that

$$
c \leqslant \min _{s \in[1, n-1] ; k \in[0, s-1]}\left\{(k+1) E X_{s-k, s+1}-k E X_{s-k, s}\right\} .
$$

By (13), all expressions in the right-hand side are positive and the above inequality is welldefined. Then, no matter how many units are supplied, and no matter how many bidders attend, it is always profitable for a seller to increase supply by one unit if he attracts one more buyer. 
By Lemma 2(3), we know that the marginal revenue is decreasing if $F$ is IFR. Then, for each $s$, the minimum in the above condition is achieved for $k=s-1$, and it is sufficient to assume that

$$
c \leqslant \min _{s \in[1, n-1]}\left\{s E X_{1, s+1}-(s-1) E X_{1, s}\right\} .
$$

Suppose now, by contradiction, that $\left(n_{1}^{*}, k_{1}^{*} ; n_{2}^{*}, k_{2}^{*}\right)$ is an equilibrium outcome, where $n_{1}^{*}, n_{2}^{*}>$ $0, n_{1}^{*}+n_{2}^{*}=n, k_{1}^{*}, k_{2}^{*}>0$ and $k_{1}^{*}+k_{2}^{*}<n$. Suppose also without loss of generality that auction 1 yields the lower expected price. Then seller 1 can increase the number of supplied units to $\left(k_{1}^{*}+1\right)$. By Lemma 2, at least one buyer will want to switch to his auction, and, by the assumption on the marginal $\operatorname{cost} c$, this constitutes a profitable deviation for seller 1 . Switching by more buyers is even more profitable since the price increases further. Therefore, $\left(n_{1}^{*}, k_{1}^{*} ; n_{2}^{*}, k_{2}^{*}\right)$ cannot be an equilibrium outcome.

Lemma 2 and condition (5) imply that a seller selling $k_{i} \leqslant \frac{n}{2}-1$ objects to $\frac{n}{2}$ bidders has incentive to increase his supply. But, if both sellers together sell $n$ objects, they cannot both make positive profits.

Proof of Proposition 5. Under condition (7) both sellers have an incentive to increase supply from $\frac{n}{2}-2$ to $\frac{n}{2}-1$, but no seller has an incentive to increase supply from $\frac{n}{2}-1$ to $\frac{n}{2}$ if by doing so only one more buyer will be attracted. If a seller sells $\frac{n}{2}-1$ units, at least $\frac{n}{2}-1$ buyers will attend his auction. Thus, no seller can attract more than one buyer by increasing the number of supplied units beyond $\frac{n}{2}-1$. Finally, no seller has an incentive to decrease supply below $\frac{n}{2}-1$ since a decrease by $k$ units induces at least $k$ buyers to switch to the other auction.

Proof of Proposition 6. (1) Assume that seller 1 sets $k_{1}=n-1$. In that case, at least $n_{1}=n-1$ will always attend auction 1, and the price in auction 1 is at most $E X_{1, n}$. Seller 2 has no way of ensuring an equal or lower price. Thus, no matter what seller 2 does, he cannot attract enough buyers, and entry is not profitable.

Assume next that seller 1 sets $k_{1}=n-2$. Given that at least $n_{1}=n-2$ buyers always attend auction 1, the only way in which seller 2 can make a profit is by auctioning one unit to 2 buyers. In that case the price is $E X_{1,2}$. By switching to auction 1, one of these two buyers will face a price of $E X_{1, n-1}<E X_{1,2}$. Thus, switching is profitable, and seller 2 can never make a profit by entry.

Continuing in this way, let $\tilde{k_{1}}$ be the minimal $k_{1}$ such that for all $k_{2}<n-\tilde{k_{1}}$, and for all $n_{2}, k_{2}<n_{2}<n-\widetilde{k_{1}}$, it holds that

$$
E X_{n_{2}-k_{2}, n_{2}}>E X_{n-n_{2}+1-\tilde{k}_{1}, n-n_{2}+1}>0 \text {. }
$$

Assume then that $k_{1}^{*} \geqslant \widetilde{k_{1}}$, and assume that selling $k_{1}^{*}$ is profitable for seller 1 (condition (8)).Then, the above inequality implies condition (9), and thus the existence of an equilibrium with a unique active site.

By Proposition 1, the assumptions in the statement are satisfied for sufficiently convex $F$ and sufficiently small $c$.

(2) Assume that $k_{1}^{*}<\frac{n}{2}$. Setting $k_{2}=k_{1}^{*}$, we obtain

$$
\begin{aligned}
E X_{n_{2}-k_{2}, n_{2}}-E X_{n-n_{2}+1-k_{1}^{*}, n-n_{2}+1} & =E X_{n_{2}-k_{1}^{*}, n_{2}}-E X_{n-n_{2}+1-k_{1}^{*}, n-n_{2}+1} \\
& =E X_{n_{2}-k_{1}^{*}, n_{2}}-E X_{n_{2}-k_{1}^{*}+\left(n-2 n_{2}+1\right), n_{2}+\left(n-2 n_{2}+1\right)}<0 .
\end{aligned}
$$

This follows because $n-2 n_{2}+1>0\left(k_{2}=k_{1}^{*}\right.$ implies $\left.n_{1}=n_{2}\right)$ and because $E X_{i, j}<E X_{i+a, j+a}$ for all $i<j$ and $a>0$. In particular, condition (9) cannot be satisfied. Thus, by replicating seller 
1's strategy, seller 2 can sustain a positive profit, and an asymmetric equilibrium with a unique active seller does not exist.

The assumption $k_{1}^{*}<\frac{n}{2}$ is satisfied if $F$ is sufficiently concave-see Proposition 1 and Corollary 2.

Proof of Proposition 7. Assume that total supply in monopoly is $2 k$ units. Denote by $g(x)=$ $\left(F^{-1}\right)^{\prime}(x)$. Then, the marginal revenue obtained by supplying an additional unit is given by

$$
\begin{aligned}
M R^{M}(k) & =(2 k+1) E X_{n-(2 k+1), n}-2 k E X_{n-2 k, n} \\
& =E X_{n-(2 k+1), n-2 k\left(E X_{n-2 k, n}-E X_{n-(2 k+1), n}\right)} \\
& \approx F^{-1}\left(\frac{n-2 k-1}{n+1}\right)-2 k\left[F^{-1}\left(\frac{n-2 k}{n+1}\right)-F^{-1}\left(\frac{n-2 k-1}{n+1}\right)\right] \\
& \approx F^{-1}\left(\frac{n-2 k-1}{n+1}\right)-\frac{2 k g\left(\frac{n-2 k}{n+1}\right)}{(n+1)} .
\end{aligned}
$$

For the competition model with one auction site and with total supply of $2 k$, the marginal revenue of a seller is

$$
\begin{aligned}
M R^{C 1}(k) & =(k+1) E X_{n-(2 k+1), n}-k E X_{n-2 k, n} \\
& =E X_{n-(2 k+1), n}-k\left(E X_{n-2 k, n}-E X_{n-(2 k+1), n}\right) \\
& \approx F^{-1}\left(\frac{n-2 k-1}{n+1}\right)-k\left[F^{-1}\left(\frac{n-2 k}{n+1}\right)-F^{-1}\left(\frac{n-2 k-1}{n+1}\right)\right] \\
& \approx F^{-1}\left(\frac{n-2 k-1}{n+1}\right)-\frac{k g\left(\frac{n-2 k}{n+1}\right)}{(n+1)} .
\end{aligned}
$$

For the competition model with two sites and total supply $2 k$, assume that a seller attracts $s$ additional bidders by increasing supply by one unit. Then his marginal revenue is

$$
M R^{C 2}(k)=(k+1) E X_{(n / 2+s)-(k+1), n / 2+s}-k E X_{n / 2-k, n / 2} .
$$

By the assumption that equilibrium supply is at least $\frac{n}{3}+2$ units, and by Lemma 9, we get that $s=1$. This yields

$$
\begin{aligned}
M R^{C 2}(k) & =(k+1) E X_{n / 2-k, n / 2+1}-k E X_{n / 2-k, n / 2} \\
& =E X_{n / 2-k, n / 2+1}-k\left(E X_{n / 2-k, n / 2}-E X_{n / 2-k, n / 2+1}\right) \\
& \approx F^{-1}\left(\frac{\frac{n}{2}-k}{\frac{n}{2}+2}\right)-k\left[F^{-1}\left(\frac{\frac{n}{2}-k}{\frac{n}{2}+1}\right)-F^{-1}\left(\frac{\frac{n}{2}-k}{\frac{n}{2}+2}\right)\right] \\
& \approx F^{-1}\left(\frac{n-2 k}{n+4}\right)-k g\left(\frac{\frac{n}{2}-k}{\frac{n}{2}+1}\right)\left(\frac{\frac{n}{2}-k}{\frac{n}{2}+1}-\frac{\frac{n}{2}-k}{\frac{n}{2}+2}\right) \\
& =F^{-1}\left(\frac{n-2 k}{n+4}\right)-\frac{k g\left(\frac{n-2 k}{n+2}\right)}{(n+2)} \frac{(n-2 k)}{\left(\frac{n}{2}+2\right)} .
\end{aligned}
$$


For large $n$ we have

$$
\begin{aligned}
& F^{-1}\left(\frac{n-2 k-1}{n+1}\right) \approx F^{-1}\left(\frac{n-2 k}{n+4}\right) \\
& \frac{k g\left(\frac{n-2 k}{n+2}\right)}{(n+2)} \approx \frac{k g\left(\frac{n-2 k}{n+1}\right)}{(n+1)} .
\end{aligned}
$$

Therefore, we obtain

$$
M R^{M}(k)<M R^{C 1}(k) \text { and } \operatorname{MR}^{M}(k)<M R^{C 2}(k) \text { for all } k^{19}
$$

On the other hand, note that

$$
\frac{(n-2 k)}{\left(\frac{n}{2}+2\right)}>1 \Leftrightarrow k<\frac{n}{4}+1 .
$$

Thus, we have

$$
\begin{cases}M R^{C 2}(k)<M R^{C 1}(k) & \text { if } k<\frac{n}{4}+1, \\ M R^{C 2}(k)>M R^{C 1}(k) & \text { if } k>\frac{n}{4}+1 .\end{cases}
$$

If the distribution $F$ has an increasing hazard rate, then by Lemma 2, the marginal revenue $M R^{C 2}(k)$ is decreasing in $k$. Note also that

$$
\begin{aligned}
& M R^{M}(k)=E X_{i, n}-(n-i+1)\left(E X_{i, n}-E X_{i-1, n}\right) \quad \text { where } i=n-2 k, \\
& M R^{C 1}(k)=\frac{1}{2} E X_{i, n}+\frac{1}{2} E X_{i-1, n}-\frac{1}{2}(n-i+1)\left(E X_{i, n}-E X_{i-1, n}\right) \text { where } i=n-2 k .
\end{aligned}
$$

By Lemma 7, both $M R^{M}(k)$ and $M R^{C 1}(k)$ are decreasing in $k$ if $F$ is IFR.

Since the marginal cost is assumed to be the same across models, the equilibrium quantity is lowest and the price is the highest under monopoly. If marginal cost is relatively low, and assuming that an equilibrium exists in the competition model with two sites, this situation is more competitive than the one with one site. The opposite occurs for high marginal cost.

When deriving the marginal revenue in the competition model with two auction sites, we assumed that the additional number of bidders that a seller can attract by increasing supply by one unit is one. The following lemma gives a mild condition under which this is indeed the case. It will be satisfied as long as $c$ is not too large, such that the equilibrium supply of each seller satisfies $k>\frac{n}{6}+1$. Analogous, weaker conditions can be derived whenever the number of attracted bidders is finite and small relative to the equilibrium quantity $k$.

Lemma 9. Consider the competition model with two auction sites (C2) for a large number of buyers $n$. Suppose that the marginal cost c satisfies

$$
c<\left(\frac{n}{6}+2\right) E X_{(n / 3-1, n / 2+1)}-\left(\frac{n}{6}+1\right) E X_{(n / 3-1, n / 2)} .
$$

Then, if a seller increases (decrease) supply by one unit, he attracts (looses) exactly one buyer.

\footnotetext{
${ }^{19}$ Note that the comparison among $M R^{M}(k)$ and $M R^{C 1}(k)$ hold always, i.e., without any additional assumptions about the number of bidders, supply, etc.
} 
Proof of Lemma 9. By the necessary condition for a symmetric equilibrium (6), and condition (14), we obtain that $k^{*}>\frac{n}{6}+1$ where $k^{*}$ denotes the equilibrium supply of each seller (otherwise, a seller always has incentive to increase quantity by one).

(1) Suppose first that seller 1 increases supply by one unit. Lemma 2 shows that he can attract at least one more bidder. Therefore, we only need to show that he cannot attract two more bidders. That is, we need to show that

$$
E X_{n / 2+2-\left(k^{*}+1\right), n / 2+2}>E X_{n / 2-1-k^{*}, n / 2-1} .
$$

For large $n$, this is equivalent to showing

$$
\begin{aligned}
F^{-1}\left(\frac{\frac{n}{2}+1-k^{*}}{\frac{n}{2}+3}\right) & >F^{-1}\left(\frac{\frac{n}{2}-1-k^{*}}{\frac{n}{2}}\right) \Leftrightarrow \frac{\frac{n}{2}+1-k^{*}}{\frac{n}{2}+3}>\frac{\frac{n}{2}-1-k^{*}}{\frac{n}{2}} \\
& \Leftrightarrow k^{*}>\frac{n}{6}+1 .
\end{aligned}
$$

(2) Suppose seller 1 decreases his supply by one. We show first that he will lose at least one bidder, and then we show that he will not lose more than one bidder.

(i) In order to show seller 1 will lose at least one bidder, it is sufficient to show

$$
E X_{n / 2-\left(k^{*}-1\right), n / 2}>E X_{n / 2+1-k^{*}, n / 2+1}
$$

which holds trivially.

(ii) In order to show seller 1 will not lose more than one bidder, it is sufficient to show

$$
E X_{(n / 2-1)-\left(k^{*}-1\right), n / 2-1}<E X_{n / 2+2-k^{*}, n / 2+2} .
$$

For large $n$, this is equivalent to

$$
F^{-1}\left(\frac{\frac{n}{2}-k^{*}}{\frac{n}{2}}\right)<F^{-1}\left(\frac{\frac{n}{2}+2-k^{*}}{\frac{n}{2}+3}\right) \Leftrightarrow \frac{\frac{n}{2}-k^{*}}{\frac{n}{2}}<\frac{\frac{n}{2}+2-k^{*}}{\frac{n}{2}+3} \Leftrightarrow k^{*}>\frac{n}{6} .
$$

\section{References}

[1] R. Amir, V.E.E. Lambson, On the effects of entry in Cournot markets, Rev. Econ. Stud. 67 (2000) $235-254$.

[2] M.R. Anderson, G. Ellison, D. Fudenberg, Location choice in two-sided markets with indivisible agents, Working paper, Harvard University, 2005.

[3] S. Anwar, R. McMillan, M. Zheng, Bidding behavior in competing auctions: evidence from eBay, Europ. Econ. Rev. 50 (2006) 307-322.

[4] K. Back, J.F. Zender, Auctions of divisible goods with endogenous supply, Econ. Lett. 73 (2001) 29-34.

[5] R. Barlow, F. Proschan, Mathematical Theory of Reliability, Wiley, New York, 1965.

[6] R. Barlow, F. Proschan, Inequalities for linear combinations of order statistics from restricted families, Ann. Math. Statist. 37 (1966) 1593-1601.

[7] R. Barlow, F. Proschan, Statistical Theory of Reliability and Life Testing, McArdle Press, Silver Spring, 1975.

[8] S. Behringer, Equilibrium market and pricing structure in virtual platform duopoly, Discussion paper, Frankfurt University, 2006.

[9] J. Bulow, P. Klemperer, Auctions versus negotiations, Amer. Econ. Rev. 86 (1) (1996) 180-194.

[10] J. Bulow, J. Roberts, The simple economics of optimal auctions, J. Polit. Economy 97 (1989) 1060-1090.

[11] R. Burguet, J. Sakovics, Imperfect competition in auction design, Int. Econ. Rev. 40 (1999) 231-247.

[12] C. Davidson, R. Deneckere, Long-run competition in capacity, short-run competition in price, and the Cournot model, RAND J. Econ. 17 (3) (1986) 404-415.

[13] G. Demange, D. Gale, M. Sotomayor, Multi-unit auctions, J. Polit. Economy 94 (1986) 863-872.

[15] G. Ellison, D. Fudenberg, Knife-edge or plateau: When do market models tip?, Quart. J. Econ. 118 (2003) 1249-1278. 
[16] G. Ellison, D. Fudenberg, M. Mobius, Competing auctions, J. Europ. Econ. Assoc. 2 (1) (2004) 30-66.

[17] D. Genesove, Price sensitivity to supply traded under alternative mechanisms in the New England fish industry, Mimeo, MIT, 1995.

[18] R. Hansen, Auctions with endogenous quantity, RAND J. Econ. 19 (1988) 44-58.

[19] H. Hoppe, B. Moldovanu, A. Sela, The theory of assortative matching based on costly signaling, Working paper, Bonn University, 2006.

[20] P. Klemperer, Markets with consumers'switching costs, Quart. J. Econ. 102 (1987) 375-394.

[21] P. Klemperer, M. Meyer, Supply function equilibria in oligopoly with demand uncertainty, Econometrica 57 (1989) 1243-1277.

[22] L. Koutsougeras, Non-Walrasian equilibria and the law of one price, J. Econ. Theory 108 (2003) 169-175.

[23] D. Kreps, J. Scheinkman, Quantity precommitment and Bertrand competition yield Cournot outcomes, Bell J. Econ. 14 (1983) 326-337.

[24] Y. Lengwiler, The multiple unit auction with variable supply, Econ. Theory 14 (1999) 373-392.

[25] M. LiCalzi, A. Pavan, Tilting the supply schedule to enhance competition in uniform price auctions, Europ. Econ. Rev. 49 (2005) 227-250.

[26] A. Matros, A. Zapechelnyuk, Optimal mechanism for an auction mediator, Discussion paper, Pittsburgh University, 2006.

[27] D. McAdams, Uniform price auctions with adjustable supply, Discussion paper, MIT, 2006.

[28] R.P. McAfee, Mechanism design by competing sellers, Econometrica 61 (1993) 1281-1312.

[29] B. Moldovanu, A. Sela, X. Shi, Contests for status, J. Polit. Economy 115 (2007) 338-363.

[30] D. Monderer, L.S. Shapley, Potential games, Games Econ. Behav. 14 (1996) 124-143.

[31] D. Monderer, M. Tenenholz, K-price auctions: revenue inequalities, utility equivalence, and competition in auction design, Econ. Theory 24 (2004) 225-270.

[32] D. Moreno, L. Ubeda, Capacity precommitment and price competition yield the Cournot outcome, Games Econ. Behav. 56 (2006) 323-332.

[33] Z. Neeman, N. Vulkan, Markets versus negotiations: the predominance of centralized markets, Mimeo, 2001.

[34] M. Peters, S. Severinov, Competition among sellers who offer auctions instead of prices, J. Econ. Theory 75 (1997) 141-179.

[35] M. Peters, S. Severinov, Internet auctions with many traders, J. Econ. Theory 130 (2006) 220-245.

[36] L. Shapley, M. Mhubik, Trade using one commodity as a means of payment, J. Polit. Economy 85 (1975) $937-968$. 